\title{
Optimized Query Planning of Continuous Aggregation Queries in Dynamic Data Dissemination Networks
}

\author{
Rajeev Gupta \\ IBM India Research Lab \\ New Delhi, India \\ grajeev@in.ibm.com
}

\author{
Krithi Ramamritham \\ Indian Institute of Technology \\ Mumbai, India \\ krithi@cse.iitb.ac.in
}

\begin{abstract}
Continuous queries are used to monitor changes to time varying data and to provide results useful for online decision making. Typically a user desires to obtain the value of some aggregation function over distributed data items, for example, to know (a) the average of temperatures sensed by a set of sensors (b) the value of index of mid-cap stocks. In these queries a client specifies a coherency requirement as part of the query. In this paper we present a low-cost, scalable technique to answer continuous aggregation queries using a content distribution network of dynamic data items. In such a network of data aggregators, each data aggregator serves a set of data items at specific coherencies. Just as various fragments of a dynamic web-page are served by one or more nodes of a content distribution network, our technique involves decomposing a client query into sub-queries and executing sub-queries on judiciously chosen data aggregators with their individual sub-query incoherency bounds. We provide a technique of getting the optimal query plan (i.e., set of subqueries and their chosen data aggregators) which satisfies client query's coherency requirement with least cost, measured in terms of the number of refresh messages sent from aggregators to the client. For estimating query execution cost, we build a continuous query cost model which can be used to estimate the number of messages required to satisfy the client specified incoherency bound. Performance results using real-world traces show that our cost based query planning leads to queries being executed using less than one third the number of messages required by existing schemes.
\end{abstract}

\section{Categories and Subject Descriptors}

H.3.5 [Online Information Services]: Web Based Services

\section{General Terms}

Algorithms, Management, Measurement, Performance, Design.

\section{Keywords}

Content distribution networks, Dynamic data, continuous aggregation queries, data coherency, query dissemination cost.

\section{INTRODUCTION}

Many data intensive applications delivered over the Web suffer from performance and scalability issues. Content distribution networks (CDNs) solved the problem for static content using

Copyright is held by the International World Wide Web Conference Committee (IW3C2). Distribution of these papers is limited to classroom use and personal use by others.

WWW 2007, May 8-12, 2007, Banff, Alberta, Canada. ACM 978-1-59593-654-7/07/0005. caches at the edge nodes of the networks. CDNs continue to evolve to serve more and more dynamic applications [1,2]. A dynamically generated web page is usually assembled using a number of static or dynamically generated fragments. The static fragments are served from the local caches whereas dynamic fragments are created either by using the cached data or by fetching the data items from the origin data sources. One important question for satisfying client requests through a network of nodes is how to select the best node(s) to satisfy the request. For static pages content requested, proximity to the client and load on the nodes are the parameters generally used to select the appropriate node [3, 4]. In dynamic CDNs, while selecting the node(s) to satisfy the client request, the central site (top-level CDN node) has to ensure that page/data served meets client's coherency requirements also. Techniques to efficiently serve fast changing data items with guaranteed incoherency bounds have been proposed in the literature $[5,6]$. Such dynamic data dissemination networks can be used to disseminate data such as stock quotes, temperature data from sensors, traffic information, and network monitoring data. In this paper we propose a method to efficiently answer aggregation queries involving such data items.

In data dissemination schemes proposed in literature $[5,6]$, a hierarchical network of data aggregators is employed such that each data aggregator serves the data item at some guaranteed incoherency bound. Incoherency of a data item at a given node is defined as the difference in value of the data item at the data source and the value at that node. Although CDNs use pagepurge [8] based coherency management, we assume that in dynamic data dissemination networks, these messages carry the new data values thereby an invalidation message becomes a refresh message. For maintaining a certain incoherency bound, a data aggregator gets data updates from the data source or some higher level data aggregator so that the data incoherency is not more than the data incoherency bound. In a hierarchical data dissemination network a higher level aggregator guarantees a tighter incoherency bound compared to a lower level aggregator. Thus data refreshes are pushed from the data sources to the clients through the network of aggregators. Dissemination networks for various data items (possibly from different data sources) can be overlaid over a single network of data aggregators as shown in Figure 1. Thus, from a data dissemination capability point of view, each data aggregator (DA) is characterized by a set of $\left(s_{i}, c_{i}\right)$ pairs, where $s_{i}$ is the data item which the DA can disseminate at an incoherency bound $c_{i}$.

Example 1: In a network of data aggregators managing data items $\mathrm{S}_{1}-\mathrm{S}_{4}$, various aggregators can be characterized as-

$D 1:\left\{\left(S_{1}, 0.5\right),\left(S_{3}, 0.2\right)\right\}$

$D 2:\left\{\left(S_{1}, 1.0\right),\left(S_{2}, 0.1\right),\left(S_{4}, 0.2\right)\right\}$ 
Aggregator D1 can serve values of $S_{1}$ with an incoherency bound greater than or equal to 0.5 whereas $D 2$ can disseminate the same data item at a looser incoherency bound of 1.0 or more. Usually, client is interested in an aggregation of these dynamic data items at a certain incoherency bound. These continuous queries are used to monitor changes in dynamic data and provide results useful for online decision making. For generating the result of a query, data from multiple sources is required. As a result, the query has to be evaluated either at data aggregators [9] or at the client. In this paper we assume existence of data dissemination network of multiple data items to answer a class of queries termed, continuous incoherency bounded weighted aggregation queries, which are formally defined next.

\subsection{Continuous Incoherency-Bounded Weighted Aggregation Queries}

A continuous weighted aggregation query can be formally written as:

$$
V_{s}^{q}(t)=\sum_{i=1}^{i=n^{q}} s_{i}(t) \times w_{i}^{q}
$$

$V_{s}^{q}$ is the value of a client query $q$ involving $n^{q}$ data items with the weight of the $i^{\text {th }}$ data item being $w^{q}{ }_{i}, 1 \leq i \leq n^{q} . s_{i}(t)$ is the value of the $i^{\text {th }}$ data item at the data source at time $t$. Such a query encompasses SQL aggregation operators SUM and AVG besides general weighted aggregation queries such as portfolio queries, involving aggregation of stock prices, weighted with number of shares of stocks in the portfolio. Due to space limitations we are not presenting execution schemes for other aggregation queries such as MIN/MAX. Interested readers are referred to [10] for the extended version of this paper.

Let the value of $i^{\text {th }}$ data item, in Equation (1), known to the client/DA be $d_{i}(t)$. Then the data incoherency is given by $\mid s_{i}(t)$ $d_{i}(t)$. For a data item which needs to be disseminated at an incoherency bound $C$ the data refresh is sent to the client or lower level DA, if the $\left|s_{i}(t)-d_{i}(t)\right|$ is more than $C$. If user specified incoherency bound for the query $q$ is $C^{q}$, then the dissemination network has to ensure that:

$$
\left|\sum_{i=1}^{n^{q}}\left(s_{i}(t)-d_{i}(t)\right) \times w_{i}^{q}\right| \leq C^{q}
$$

Whenever data values at sources change such that query incoherency bound is violated, the updated value(s) is disseminated to the client. If the network of aggregators can ensure that the $i^{\text {th }}$ data item has incoherency bound $C_{i}$ then the following condition ensures that the query incoherency bound $C^{q}$ is satisfied:

$$
\sum_{i=1}^{n^{q}} C_{i} \times w_{i}^{q} \leq C^{q}
$$

A client specified query incoherency bound needs to be translated into incoherency bounds for individual data items or sub-queries such that Equation (3) is satisfied. It should be noted that Equation (3) is sufficient condition for satisfying the query incoherency bound but not the necessary. This way of translating the query incoherency bound into the sub-query incoherency bounds is required if data is transferred between various nodes using only push based mechanism. Such a translation is not required in either a pull based mechanism as shown in our earlier paper [9] or combinations of push and pull. In this paper we consider only push based data dissemination among servers, DAs and clients. Next we present the summary of our approach towards executing the continuous multi-data weighted additive aggregation query with the objective of minimization of number of refreshes from data aggregators to the client. Our technique can be used for various popular applications where different clients require aggregation of multiple data items at their individual incoherency bounds. Monitoring stock portfolios is one such popular application which we use for performance measurements.

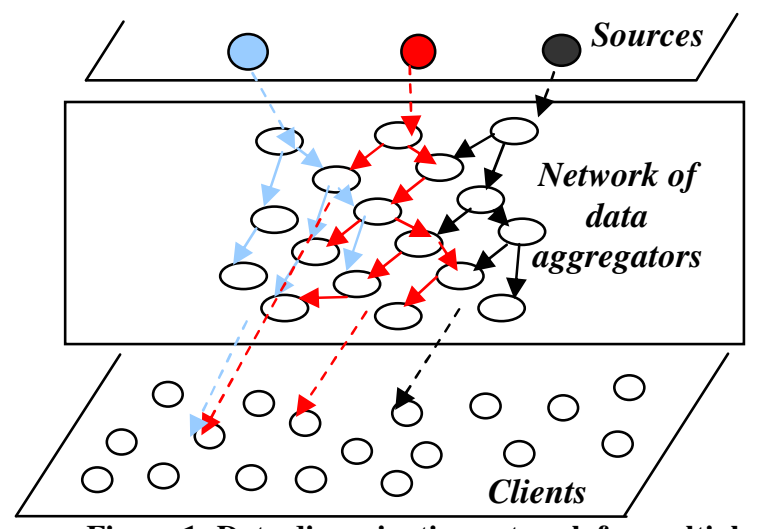

Figure 1: Data dissemination network for multiple data items

\subsection{Summary of Approach and Contributions}

Consider a client query $Q 1=50 S_{1}+200 S_{2}+150 S_{3}$ with a required incoherency bound of 80 (in a stock portfolio $S_{1}, S_{2}, S_{3}$ can be different stocks and incoherency bound can be $\$ 80$ ). We want to execute this query over data aggregators given in Example1 minimizing number of refreshes. There are various options for the client to get the data items:

- The client may get the data items $S_{1}, S_{2}$ and $S_{3}$ separately. The query incoherency bounds can be divided among data items in various ways while satisfying Equation 3. In this paper, we show that getting data items independently is a costly option. This strategy ignores facts that the client is interested only in the aggregated value of the data items and various aggregators can disseminate more than one data item.

- If a single DA can disseminate all three data items required to answer the client query, the DA can construct a composite data item corresponding to the client query $\left(S_{q}=50 S_{1}+200 S_{2}\right.$ $+150 S_{3}$ ) and disseminate the result to the client so that the query incoherency bound is not violated. It is obvious that if we get the query result from a single DA, the number of refreshes will be minimum (as in this case data item updates may cancel out each other, thereby keeping the query result within the incoherency bound). As different data aggregators disseminate different subsets of data items, no data aggregator may have all the data items required to execute the client query which is indeed the case in Example1. Further, even if an aggregator can disseminate all the data items, it may not be able to satisfy the query coherency requirements. In such cases the query has to be executed with data from multiple aggregators.

- Another option is to divide the query into a number of subqueries and get their values from individual DAs. In that case, the client query result is obtained by combining the results of 
more than one sub-query. For the DAs given in Example1, the query $Q 1$ can be divided in two alternative ways:

plan1: D1 $\left\{50 S_{1}+150 S_{3}\right\} ; D 2\left\{S_{2}\right\}$

plan2: D1 $\left\{S_{3}\right\} ; D 2\left\{50 S_{1},+200 S_{2}\right\}$

i.e., in plan1 result of sub-query $50 S_{1}+150 S_{3}$ is disseminated by $D 1$ and that of $S_{2}$ (or $200 S_{2}$ ) by $D 2$. Combining them at the client gives the query result.

- Selecting the optimal plan among various options is not-trivial. As a thumb-rule, we should be selecting the plan with lesser number of sub-queries. But that is not guaranteed to be the plan with the least number of messages. Further, we should select the sub-queries such that updates to various data items appearing in a sub-query have more chances of canceling each other as that will reduce the need for refresh to the client (Equation 2). In the above example, if updates to $S_{I}$ and $S_{3}$ are such that when $S_{1}$ increases, $S_{3}$ decreases, and vice-versa, then selecting plan1 may be beneficial. We give an algorithm to select the query plan based on these observations.

- While solving the above problem of selecting the optimal plan we ensure that each data item for a client query is disseminated by one and only one data aggregator. Although a query can be divided in such a way that a single data item is served by multiple DAs (e.g., $50 S_{1}+200 S_{2}+150 S_{3}$ is divided into two sub-queries $50 S_{1}+130 S_{2}$ and $70 S_{2}+150$ $S_{3}$ ); but in doing so the same data item needs to be processed at multiple aggregators, increasing the unnecessary processing load. By dividing the client query into disjoint sub-queries we ensure that a data item update is processed only once for each query (For example, in case of paid data subscriptions it is not prudent to get the same data item from the multiple sources).

- The query incoherency bound needs to be divided among subquery incoherency bounds such that, besides satisfying the client coherency requirements, the chosen DA (where the subquery is to be executed) is capable of satisfying the allocated sub-query incoherency bound. For example, in plan1 allocated incoherency bound to the sub-query $50 S_{1}+150 S_{3}$ should be greater than $55(=50 * 0.5+150 * 0.2)$ as that is the tightest incoherency bound which the aggregator $D 1$ can satisfy. We prove that the number of refreshes depends on the division of the query incoherency bounds among sub-query incoherency bounds. Similar result was proved for data incoherency bounds in [11].

Thus, what we need is a method of (a) optimally dividing client query into sub-queries and (b) assigning incoherency bounds to them; such that (c) selected sub-queries can be executed at chosen DAs and (d) total query execution cost, in terms of number of refreshes, is minimized. We prove that the problem of choosing sub-queries while minimizing query execution cost is an $N P$ hard problem. We give efficient approximation algorithms to choose the set of sub-queries and their corresponding incoherency bounds for a given client query. In contrast, all related work in this area $[11,12]$ propose getting individual data items from the aggregators which, as we show in this paper, leads to large number of refreshes. For solving the above problem of optimally dividing the client query into sub-queries, we first need a method to estimate query execution cost for various alternative options. A method for estimating the query execution cost is another important contribution of this paper. As we divide the client query into sub-queries such that each sub-query gets executed at different aggregator nodes, the query execution cost (i.e., number of refreshes) is the sum of the execution costs of its constituent sub-queries. We model the sub-query execution cost as a function of following parameters:

(a) Dissemination costs of the individual data items involved. The data dissemination cost is dependent on data dynamics and incoherency bound associated with the data. We model the data dynamics using a data synopsis model, and the effect of the incoherency bound using an incoherency bound model. These two models are combined to get the estimate of the data dissemination cost.

(b) A correlation measure of data dynamics, quantifying the chance that the updates of two data items will cancel each other out such that a sub-query of their sum will incur less refreshes than disseminating the individual data changes. We use cosine similarity between data items for this purpose. This parameter is widely used in information retrieval domain [20].

Through extensive simulations we show that:

- Our method of dividing query into sub-queries and executing them at individual DAs requires less than one third of the number of refreshes required in the existing schemes.

- For efficient execution, more dynamic data item should be part of sub-query involving larger number of data items.

Our method of executing queries over dynamic data dissemination network is practical since it can be implemented using a mechanism similar to URL-rewriting [4] in CDNs. Just like in a $\mathrm{CDN}$, the client sends its query to the central site. For getting appropriate aggregators (edge nodes) to answer the client query (web page), the central site has to first determine which data aggregators have the data items required for the client query. If the client query can not be answered by a single data aggregator, the query is divided into sub-queries (fragments) and each subquery is assigned to a single data aggregator. In case of a CDN, web page's division into fragments is a page design issue, whereas, for continuous aggregation queries, this issue has to be handled on per-query basis by considering data dissemination capabilities of data aggregators as represented in Example 1.

\subsection{Outline of the Paper}

We give a formal mathematical definition of the query plan selection problem in Section 2. Query cost model for a multi-data incoherency bounded aggregation query is developed in Section 3. The query cost model uses the data dissemination model presented in Section 3.1 and cosine similarity measure which is explained in Section 3.2. In Section 4, we first prove that the optimization problem presented in Section 2 is NP-hard then we give approximate algorithms for the problem. In Section 5, performance evaluation done using real-world traces is presented to show that our sub-query based query evaluation scheme executes the client query at less than one third cost compared to other known schemes. Related work is presented in Section 6 and the paper concludes in Section 7.

\section{QUERY PLAN SELECTION PROBLEM}

In this section, we give a formal definition of the optimization problem described in the previous section. We are given a set $D$ of data aggregators, set $S$ of data items and one-to-many mapping $f$ : $D \rightarrow(S, C)$ where $C \subset \Re$ is a sub-set of real number representing incoherency bounds for various data items (in the set $S$ ) at 
aggregators in $D$. Each incoming client query $q$ over the data items set $S^{q} \subseteq S$ has corresponding weights given as a set $W^{q}$. Thus the query can be represented as set of tuples of $\langle$ data_item, weight $\rangle$, i.e., $q=\left\{\left\langle s^{q}, w^{q}\right\rangle\right\}$ with the query incoherency bound $C^{q}$. We need to perform the following two tasks such that the number of refreshes to the client is minimum:

Task1: Divide the client query $q=\left\{\left\langle s^{q}, w^{q}\right\rangle\right\}$ into sub-queries $q_{k}=$ $\left\{\left\langle s_{k}^{q}, w_{k}^{q}\right\rangle\right\}$ so that $\cup q_{k}=q$ i.e., although different sub-queries may be executed at different aggregators, combining their results gives the value of the client query.

Task2: Allocate each sub-query $q_{k}$, with its incoherency bound $C_{k}$, to data aggregators.

While fulfilling the following conditions:

Condition1: Query incoherency bound is satisfied, i.e., $\sum_{k} C_{k} \leq C^{q}$.

The sub-query $q_{k}$ should be assigned to a data aggregator $d_{i} \in D$ iff:

Condition2: The chosen aggregator should have all the data items appearing in the sub-query i.e. $\Pi_{S}\left(q_{k}\right) \subseteq \Pi_{S}\left(f\left(d_{i}\right)\right)$. Here $\Pi$ indicates project operator in relational algebra.

Condition3: Data incoherency bounds at the selected data aggregator $c_{j}=\prod_{C}\left(\sigma_{s=s_{k}^{q}(j)}\left(f\left(d_{i}\right)\right)\right.$ should be such that $C_{k} \geq X_{k}$ where $s_{k}^{q}(j)$ is the $j^{\text {th }}$ data item appearing in the subquery $q_{k}$ and $X_{k}$ is the tightest incoherency bound the aggregator can ensure for the given sub-query. $X_{k}$ can be calculated as:

$X_{k}=\sum c_{j} w_{j}^{q}$. Here $\sigma$ indicates select operator in relational algebra.

\section{QUERY COST MODEL}

Before developing the query cost model we first summarize the model to estimate the number of refreshes required to disseminate a data item at certain incoherency bound. For simulation experiments we use data items from sensor network and stock data domains as explained in our previous work [9]. Stock traces of 45 stocks were obtained by periodically polling http://finance.yahoo.com. Sensor network data used were temperature and wind sensor data from Georges Bank Cruises Albatross Shipboard [13]. Due to paucity of space we present results using stock data only but similar results were obtained for sensor data as well [14]. For detailed analysis and simulation results, readers can refer to the extended version of the paper [10].

\subsection{Data Dissemination Cost}

Cost of disseminating a data item at a certain given incoherency bound $C$ can be estimated by combining two models:

- Incoherency bound model is used for estimating dependency of data dissemination cost over the desired incoherency bound. As per this model, we have shown in [10] that the number of data refreshes is inversely proportional to the square of the incoherency bound $\left(1 / C^{2}\right)$. Similar result was earlier reported in [5] where the data dynamics was modeled as a randomwalk process.

$$
\text { Data dissemination cost } \propto 1 / C^{2}
$$

- Data Synopsis Model is used for estimating the effect of data dynamics on number of data refreshes. We define a data dynamics measure called, sumdiff, to obtain a synopsis of the data for predicting the dissemination cost. The number of update messages for a data item is likely to be higher if the data item changes more in a given time window. Thus we hypothesize that cost of data dissemination for a data item will be proportional to sumdiff, defined as:

$$
R_{s}=\sum_{i}\left|s_{i}-s_{i-1}\right|
$$

where $s_{i}$ and $s_{i-1}$ are the sampled values of the data item at $i^{\text {th }}$ and $(i-1)^{\text {th }}$ time instances (consecutive ticks). In [10] we corroborate the above hypothesis using simulation over a large number of data items. Pearson product moment correlation coefficient (PPMCC) [19] values, used for quantifying linearity between data sumdiff and number of refreshes required to maintain a fixed incoherency bound, were found to be between 0.90 and 0.96 for various values of incoherency bounds. Sumdiff value for a data item can be calculated at the data source by taking running average of difference between data values at the consecutive ticks. A data aggregator can also estimate the sumdiff value by interpolating the disseminated values.

Thus, the estimated dissemination cost for data item $S$, disseminated with an incoherency bound $C$, is proportional to $R_{s} / C^{2}$. Next we use this result for developing the query cost model.

\subsection{Query Dissemination Cost}

Consider a case where a query consists of two data items $P$ and $Q$ with weights $w_{p}$ and $w_{q}$ respectively; and we want to estimate its dissemination cost. If data items are disseminated separately, the query sumdiff will be:

$R_{\text {data }}=w_{p} R_{p}+w_{q} R_{q}=w_{p} \sum\left|p_{i}-p_{i-1}\right|+w_{q} \sum\left|q_{i}-q_{i-1}\right|(6)$

Instead, if the aggregator uses the information that client is interested in a query over $P$ and $Q$ (rather than their individual values), it makes a composite data item $w_{p} p+w_{q} q$ and disseminates that data item then the query sumdiff will be:

$$
R_{\text {query }}=\sum\left|w_{p}\left(p_{i}-p_{i-1}\right)+w_{q}\left(q_{i}-q_{i-1}\right)\right|
$$

$R_{\text {query }}$ is clearly less than or equal compared to $R_{\text {data }}$. Thus we need to estimate the sumdiff of an aggregation query (i.e., $R_{\text {query }}$ ) given the sumdiff values of individual data items (i.e., $R_{p}$ and $R_{q}$ ). Only data aggregators are in position to calculate $R_{\text {query }}$ as different data items may be from different sources. We develop the query dissemination model in two stages.

\subsubsection{Quantifying correlation between dynamics of data}

From Equations (6) and (7) we can see that if two data items are correlated such that if value of one data item increases, that of the other data item also increases, then $R_{\text {query }}$ will be closer to $R_{\text {data }}$ whereas if the data items are inversely correlated then $R_{\text {query }}$ will be less compared to $R_{\text {data }}$. Thus, intuitively, we can represent the relationship between $R_{\text {query }}$ and sumdiff values of the individual data items using a correlation measure associated with the pair of data items. Specifically, if $\rho$ is the correlation measure then $R_{\text {query }}$ can be written as:

$$
R_{\text {query }}^{2} \propto\left(w_{p}^{2} R_{p}^{2}+w_{q}^{2} R_{q}^{2}+2 \rho w_{p} R_{p} w_{q} R_{q}\right)
$$


The correlation measure is defined such that $-1 \leq \rho \leq+1$, so, $R_{\text {query }}$ will always be less than $\left|w_{p} R_{p}+w_{q} R_{q}\right|$ (as explained earlier) and always be more than $\left|w_{p} R_{p}-w_{q} R_{q}\right|$. The correlation measure $\rho$ can be interpreted as cosine similarity [20] between two streams represented by data items $P$ and $Q$. Cosine similarity is a widely used measure in information retrieval domain where documents are represented using a vector-space model and document similarity is measured using cosine of angle between two document representations. For data streams $P$ and $Q, \rho$ can be calculated as:

$$
\rho=\frac{\sum\left(p_{i}-p_{i-1}\right)\left(q_{i}-q_{i-1}\right)}{\sqrt{\sum\left(p_{i}-p_{i-1}\right)^{2}} \sqrt{\sum\left(q_{i}-q_{i-1}\right)^{2}}}
$$

\subsubsection{Query normalization}

Suppose we want to compare the cost of two queries: a SUM query involving two data items and an AVG query involving the same data items. Let the query incoherency bound for the SUM and the AVG queries be $C_{1}=2 C$ and $C_{2}=C$, respectively. From Equation (8), sumdiff of the SUM query will be double that of the AVG query (as the weight of each data item in the SUM query is double of that in the AVG query). Hence, query evaluation cost (as per $R_{i} / C_{i}^{2}$ ) of the SUM query will be half that of the AVG query (as SUM query incoherency bound is double). But, intuitively, disseminating the SUM of two data items, at double the incoherency bound should require the same number of messages as their AVG. Thus, there is a need to normalize query costs. From a query execution cost point of view, a query with weights $w_{i}$ and incoherency bound $C$ is same as query with weights $a w_{i}$ and incoherency bound a.C. So, while normalizing we need to ensure that both, query weights and incoherency bounds, are multiplied by the same factor. Normalized query sumdiff is given by:

$$
R_{\text {query }}^{2}=\frac{\left(w_{p}^{2} R_{p}^{2}+w_{q}^{2} R_{q}^{2}+2 \rho w_{p} R_{p} w_{q} R_{q}\right)}{\left(w_{p}^{2}+w_{q}^{2}+2 \rho w_{p} w_{q}\right)}
$$

i.e., the value of the normalizing factor should be $1 / \sqrt{w_{p}^{2}+w_{p}^{2}+2 \rho w_{p} w_{q}}$. The value of the incoherency bound has to be adjusted by the same factor. Normalization ensures that queries with arbitrary values of weights can be compared for execution cost estimates. From Equations (9 and 10) the value of query sumdiff can be estimated at a data aggregator node if it has all the required data items disseminated to it. An aggregator can use interpolated values of data items to estimate $\rho$ as it is not (always) likely to have all the data updates. In the extended version of the paper [10] we present an efficient method (using [21]) to calculate $\rho$ which can also be used when the corresponding data items are not being disseminated by the same data aggregator. Equation (10) can be extended to get query sumdiff for any general weighted aggregation query given by Equation (1) as:

$$
R_{Q}^{2}=\frac{\sum_{i=1}^{n} w_{i}^{2} R_{i}^{2}+2 \sum_{i=1}^{n} \sum_{j=1, j \neq i}^{n} \rho_{i j} w_{i} w_{j} R_{i} R_{j}}{\sum_{i=1}^{n} w_{i}^{2}+2 \sum_{i=1}^{n} \sum_{j=1, j \neq i}^{n} \rho_{i j} w_{i} w_{j}}
$$

\subsubsection{Validating the query cost model}

To validate the query cost model we performed simulations by constructing more than 50 weighted aggregation queries using the stock data with each query consisting of 3-7 data items with data weights uniformly distributed between 1 and 10 . For each query the number of refreshes was counted for various normalized incoherency bounds between 0.01 and 0.5. Figure 2 shows that the number of messages is proportional to the normalized query sumdiff as calculated using Equation (11) if their normalized incoherency bounds are same. In this case PPMCC value is found to be $95 \%$. Similarly, Figure 3 shows the dependence of the number of refreshes on $1 / C^{2}$ to prove that the relationship that holds between them for single data item also holds for a query with multiple data items. The query cost model can be used in various applications of query assignment, load balancing, optimal order of processing, etc. In the next section, we use this query cost model for our query plan problem to optimally divide a client query into sub-queries and execute it over a network of data aggregators so that the number of refreshes can be minimized

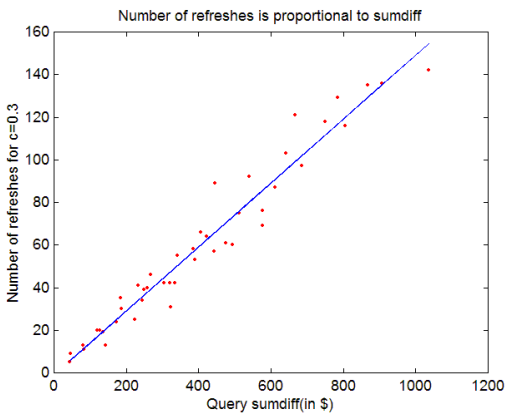

Figure 2: Variation of query cost with query sumdiff (Normalized $C=0.3$ )

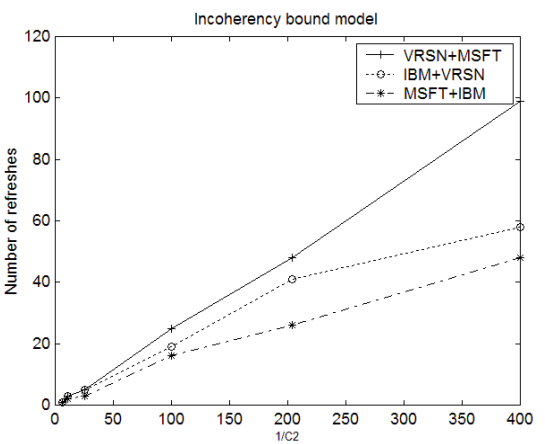

Figure 3: Number of refreshes for varying query incoherency bounds

\section{EXCEUCTING QUERIES USING SUB- QUERIES}

For executing an incoherency bounded continuous query, a query plan is required which includes the set of sub-queries, their individual incoherency bounds and data aggregators which can execute these sub-queries. We need to find the optimal query execution plan which satisfies client coherency requirement with the least number of refreshes. As explained in Section 1, what we need is a mechanism to:

Task 1: Divide the aggregation query into sub-queries; and 
Task 2: Allocate the query incoherency bound among them.

while satisfying the following conditions identified in Section 2:

condition 1. Query incoherency bound is satisfied.

condition 2. The chosen DA should be able to provide all the data items appearing in the sub-query assigned to it.

condition 3. Data incoherency bounds at the chosen DA should be such that the sub-query incoherency bound can be satisfied at the chosen DA.

Objective : Number of refreshes should be minimized.

Let the client query be divided into $N$ sub-queries $\left\{q_{k}: 1 \leq k \leq N\right\}$; with $R_{k}$ being sumdiff of $k^{\text {th }}$ sub-query and $C_{k}$ being incoherency bound assigned to it. As given is Section 3, the dissemination cost of a sub-query is estimated to be proportional to $R_{k} / C_{k}^{2}$. Thus query cost estimate is given by:

$$
Z=\sum_{k=1}^{N}\left(R_{k} / C_{k}^{2}\right)
$$

While allocating sub-query incoherency bounds we need to ensure that the query coherency requirement $C$ is satisfied (condition1); i.e.,

$$
\sum_{k=1}^{N} C_{k} \leq C
$$

For satisfying condition2, sub-queries should be such that all its data items can be disseminated by the chosen DA. Let $X_{k}$ be the tightest incoherency bound (defined in Section 2) the chosen DA can satisfy for $q_{k}$. For the condition3, we have to ensure that $C_{k} \geq$ $X_{k}$ for each sub-query $q_{k}$ and its assigned data aggregator. $Z$ needs to be minimized for minimizing the number of refreshes as per the objective.

Before attempting the hard problem of optimizing $Z$, let us first consider a simpler problem where values of $C_{k}$ are given. In this simpler problem we divide the client query into sub-queries to minimize the estimated execution $\operatorname{cost}(Z)$ without considering the optimal division of the query incoherency bound into sub-query incoherency bounds. Besides working as a step towards a solution for the whole problem this case can also be used where allocation of incoherency bounds to sub-queries is done independent of the data dynamics. For example, it may be pre-decided that incoherency bounds for all data items will be the same. Thus, for a given query and its incoherency bounds, the sub-query incoherency bounds can be obtained. Next we prove that this simpler version of the problem is NP-hard.

\subsection{Finding Optimal Query Plan is NP-hard}

For proving that the problem is NP-hard, we use reduction from 3-dimensional matching (3DM) problem. For a given client query and DA, let us first define maximal sub-query as the largest part of the query which can be disseminated by the DA (i.e., the maximal sub-query has all the query data items which the DA can disseminate at the required incoherency bound). For example, for a client query $20 S_{1}+25 S_{2}+35 S_{3}$ with incoherency bound 80 ; let the pre-decided incoherency bound for each data item be 1 . For the data aggregators $D 1$ and $D 2$ given in Example 1, the maximal sub-query for $D 1$ will be $q_{1}=20 S_{1}+35 S_{3}$, whereas for $D 2$ it will be $q_{2}=20 S_{1}+25 S_{2}$.
3DM Problem: Given three sets $X, Y$ and $Z$, each with $N$ elements, and a set $M \subseteq X \times Y \times Z$, is there a subset $M I \subseteq M$ such that $|M 1|=N$ and no two elements of $M 1$ agree in any coordinate?

We use a slightly different (decision) version of the optimization problem to reduce the $3 \mathrm{DM}$ problem. To solve the $3 \mathrm{DM}$ problem we reduce it to a SUM query of $3 N$ items:

- The SUM query: $\sum_{i=1}^{N}\left(x_{i}+y_{i}+z_{i}\right)$ for $x_{i} \in X, y_{i} \in Y, z_{i} \in Z$.

- We assume that all the data items have the same sumdiff values of 1 ; cosine similarity between all the data items is 0 ; and all data items are allocated an incoherency bound of 1 .

- For each element $\left(x_{i}, y_{j}, z_{k}\right) \in \mathrm{M}$, we assume the existence of a data aggregator disseminating these three data items only.

- In the decision version of optimal plan problem we ask whether there exists a query plan with query cost estimate value $N / 3$.

If a query plan with cost estimate value $N / 3$ exists; it implies that the query plan has $\mathrm{N}$ queries with 3 items each (that will lead to query cost value of $1 / 3$ per sub-query as per Equation (11) whereas any other combination of sub-queries will lead to more cost). Three data items from each chosen data aggregators form a triplet for the set $M 1$ which solves 3DM. Because of space constraints we are not giving the complete proof of NPhardness of the original problem. In general, there is no known approximate algorithm for such a problem. It should be noted that performing Taskl for achieving the objective is NP-hard, so we give two greedy heuristics in next two sub-sections; whereas Task 2 can be performed optimally with conditions $1-3$ while achieving the objective. In our approach, we first try to perform Task1, while satisfying as many conditions as possible, and then optimally perform Task 2 while satisfying all the conditions.

\subsection{Minimum Cost Heuristic}

Figure 4 shows the outline of greedy heuristics where different criteria $(\psi)$ can be used to select sub-queries. In this section we describe the case where the estimate of query execution cost is minimized in each step of the algorithm (min-cost) whereas in the next section we present the case where gain due to executing a query using sub-queries is maximized (max-gain).

\subsubsection{Query Plan with Pre-decided Incoherency Bound Allocation}

For the given client query $(q)$ and mapping between data aggregators and the corresponding \{data-item, data incoherency bound $\}$ pairs $(f: D \rightarrow(S, C))$ maximal sub-queries can be obtained for each data aggregator. Let $A$ be the set of such maximal subqueries. In this set, each query $a \in A$ can be disseminated by a designated data aggregator at the assigned incoherency bound. For each sub-query $a \in A$, its Sumdiff $R_{a}$ is calculated using Equation 11. Using the set $A$ and sub-query sumdiffs, we use the algorithm outlined in Figure 4 to get the set of sub-queries minimizing the query cost. In this Figure each sub-query $a \in A$ is represented by the set of data items covered by it. As we need to minimize the query cost, a sub-query with minimum cost per data item is chosen in each iteration of the algorithm i.e., criteria $\psi \equiv$ minimize $\left(R_{a} / C_{a}{ }^{2}|a|\right)$. All data items covered by the selected subquery are removed from all the remaining sub-queries in $A$ before performing the next iteration. 


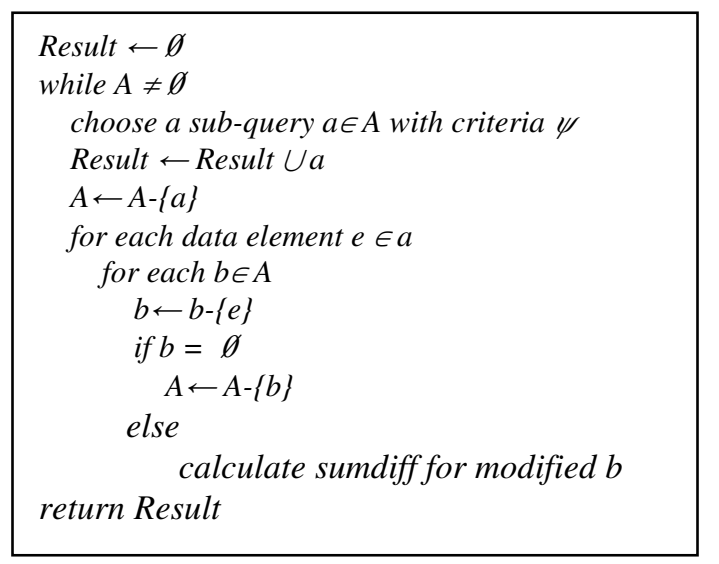

Figure 4: Greedy algorithm for query plan selection

\subsubsection{Optimizing query execution cost}

Now we consider the overall problem to select the optimal set of sub-queries while simultaneously dividing the query incoherency bound among them. In this case we get the set of maximal queries (A) without considering the minimum incoherency bounds that the data aggregators can satisfy (i.e., condition3). In this algorithm we first get the optimal set of sub-quires without considering the condition 3 and then allocate incoherency bound among them using condition1 (Equation (13)) and condition3. Lagrange multiplier scheme can be used to solve for incoherency bounds (from Equations $12 \& 13$ ) so that $Z$ is minimized:

$$
C_{k}=R_{k}^{1 / 3} C /\left(\sum_{k=1}^{N} R_{k}^{1 / 3}\right)
$$

i.e., without the constraints of condition 3 , sub-query incoherency bounds should be allocated in proportion to $R_{k}^{1 / 3}$. Using Equations (12) and (14) we get:

$$
Z^{1 / 3}=\frac{1}{C^{2 / 3}} \sum_{k=1}^{N} R_{k}^{1 / 3}
$$

From Equation (15), it is clear that for minimizing the query execution cost we should select the set of sub-queries so that $\sum R_{k}^{1 / 3}$ is minimized. We can do that by using criteria $\psi \equiv$ minimize $\left(R_{a}^{1 / 3} \wedge a \mathrm{l}\right)$ in the algorithm described in Figure 4. Once we get the optimal set of sub-queries we can use the Equation (13) and condition $3\left(C_{k} \geq X_{k}\right)$ to optimally allocate the query incoherency bound among them. This allocation problem can be solved by various convex optimization techniques available in the literature such as gradient descent method, barrier method etc. We used gradient descent method (fmincon function in MATLAB) to solve this non-linear optimization problem to get the values of individual sub-query incoherency bounds. But this method of first selecting sub-queries and then allocating the incoherency bounds has a problem which is described next.

\subsubsection{Satisfiability of Condition 3}

In the solution described in the previous section, we select the set of sub-queries (and corresponding DAs) and then allocate the query incoherency bound among them using convex optimization techniques. But the problem of incoherency bound allocation among chosen DAs may not have any feasible solution. There may be situations where, although the data dissemination network is able to satisfy the query coherency requirements but once the set of sub-queries (and corresponding DAs) is selected the incoherency bound allocation is not possible.

Example 2: Consider a client query $50 S_{1}+200 S_{2}+150 S_{3}$ with the incoherency bound of 80 and data dissemination network consisting of two aggregators $D 1$ and $D 2$ as given in Example 1. There are (at-least) two possible query plans to answer the above query:

Plan1: D1 $\left(50 S_{1}+150 S_{3}\right) ; D 2\left(S_{2}\right)$

Plan2: D1 $\left(S_{3}\right) ; D 2\left(50 S_{1}+200 S_{2}\right)$

In Section 4.2.2 we are selecting sub-queries having minimum $\sum R_{k}^{1 / 3}$, thus based on data dynamics it is possible that we select plan2 as the optimal plan. But from the data incoherency bounds that aggregators $D 1$ and $D 2$ can ensure, we see that it is not possible for plan2 to satisfy the client specified incoherency bound as minimum incoherency bound that can be satisfied by the selected aggregators $(X=50 * 1+200 * 0.1+150 * 0.2$ $=100)$ is greater than the query incoherency bound $(=80)$. Thus although there exists a plan (plan1) which can satisfy the client query incoherency bound, while minimizing the query execution cost the above method cannot ensure that such a plan will be selected.

What we need is a compromise between the query satisfiability and performance. In Section 4.2.2 we are selecting the sub-queries without considering the data incoherency bounds for the selected data aggregators. We correct that by selecting sub-queries using $\sum\left(R_{a}^{1 / 3}+\frac{\alpha X_{a}}{C R_{a}^{1 / 3}}\right)$ as substitute objective function instead of $\sum R_{a}^{1 / 3}$. The second term ensures that while selecting the optimal plan we prefer the data aggregators having tighter data incoherency bounds (lower values of $X_{a}$ ) thus higher chances of satisfying the query. The tuning parameter $(\alpha)$ can used to balance the objectives of minimizing query execution cost through subqueries selection and meeting the query coherency requirements. We use $X_{a} / C R_{a}^{1 / 3}$ in the second term as, according to Equation (14), optimal incoherency bound allocation is likely to be done proportional to $C R_{a}^{1 / 3}$. In Section 5.2, we measure effects of the tuning parameter $\alpha$ on the query satisfiability.

\subsection{Maximum Gain Heuristic}

In this section we present an algorithm which, instead of minimizing the estimated query execution cost, maximizes the estimated gains of executing client query using sub-queries. In this algorithm, for each sub-query, we calculate the relative gain of executing it by finding the sumdiff difference between cases when each data item is obtained separately and when all the data items are aggregated as a single sub-query. Thus, the relative gain for a sub-query $w_{p} p+w_{q} q$ can be written as:

$$
G_{\text {query }}=\frac{\left(w_{p} R_{p}+w_{q} R_{q}\right)}{\sqrt{\left(w_{p}^{2} R_{p}^{2}+w_{q}^{2} R_{q}^{2}+2 \rho w_{p} w_{q} R_{p} R_{q}\right)}}-1
$$

This algorithm can be implemented by using criteria $\psi \equiv$ maximize $\left(G_{\text {query }} /|a|\right)$ to get the set of sub-queries and corresponding DAs. Then we use the convex optimization method outlined in Section 4.2 to allocate incoherency bounds among sub-queries. To tackle the query satisfiability issue the query gain Equation (16) is modified to: 


$$
G_{\text {query }}^{\prime}=G_{\text {query }}-\frac{\alpha\left(w_{p} X_{p}+w_{q} X_{q}\right)}{C R_{\text {query }}^{1 / 3}}
$$

where $X_{p}$ is minimum incoherency bound that can be satisfied for the data item $P$; $C$ is query incoherency bound and $R_{\text {query }}$ is the query sumdiff $\left(=\sqrt{\left(w_{p}^{2} R_{p}^{2}+w_{q}^{2} R_{q}^{2}+2 \rho w_{p} w_{q} R_{p} R_{q}\right)}\right)$. Reasons for selecting the particular substitute objective function are same as ones outlined in Section 4.2.3. In the next Section, through performance results, we show that this algorithm performs better than the min-cost heuristic.

\section{PERFORMANCE EVALUATION}

For performance evaluation we simulated the data dissemination networks of 25 stock data items over 25 aggregator nodes such that each aggregator can disseminate combinations of up to 10 data items with data incoherency bounds chosen uniformly between $\$ 0.005$ and 0.02 . Then we created 500 portfolio queries such that each query has up to 10 randomly (uniformly) selected data items with weights varying between 2 and 10 . These queries were executed with incoherency bounds between 0.3 and 1.0 (i.e., $0.03-0.1 \%$ of the query value). In the first set of experiments, we kept the data incoherency bounds at the data aggregators very low so that query satisfiability can be ensured.

\subsection{Comparison of algorithms}

For comparison with our algorithms, presented in the previous section, we consider various other query plan options. Each query can be executed by disseminating individual data items or by getting sub-query values from DAs. Set of sub-queries can be selected using sumdiff based approaches or any other random selection. Sub-query (or data) incoherency bound can either be pre-decided or optimally allocated. Various combinations of these dimensions are covered in the following algorithms:

1. No sub-query, equal data incoherency bound (naïve): In this algorithm, the client query is executed with each data item being disseminated independent of other data items in the query. Incoherency bound is divided equally among the data items. This algorithm acts as a baseline algorithm.

2. No sub-query, optimal incoherency bound (optc): In this algorithm also data items are disseminated separately but incoherency bound is divided among data items so that total number of refreshes can be minimized. This algorithm is similar to the one presented in [11]. Here, the incoherency bound is allocated dynamically using Equation (14).

3. Random sub-query selection (random): In this case, subqueries are generated by randomly selecting one data aggregators and allocating it the maximal sub-query consisting of query data items which the aggregator can disseminate. Then the process is repeated for the remaining data items until the whole query is covered. This algorithm is designed to see how the sub-query selection based on query sumdiff (Section 4) works in comparison to random selection of sub-queries.

4. Sub-query selection while minimizing sumdiff (min-cost): This algorithm is described in Section 4.2.

5. Sub-query selection while maximizing gain (max-gain): This algorithm is described in Section 4.3.
Figure 5 shows average number of refreshes required for query incoherency bounds of $\$ 0.3, \$ 0.5$ and $\$ 0.8$. The naïve algorithm requires more than three times the number of messages compared to min-cost and max-gain algorithms. For incoherency bound of $\$ 0.8$ each query requires 1024 messages if it is executed just by optimizing incoherency bound (optc) compared to 255 when we select the query plan using the max-gain algorithm. Further, although the optimization problem is similar to the covering a set of data items (query) using its sub-sets (sub-queries) for which the greedy min-cost algorithm is considered to be most efficient [7], we see that max-gain algorithm requires $20-25 \%$ less messages compared to the min-cost approach. Reasons for max-gain algorithm performing better than other algorithms are explored in the next set of experiments. Although here we presented results for stock traces (man-made data) similar results were obtained for sensor traces (natural data) as well.

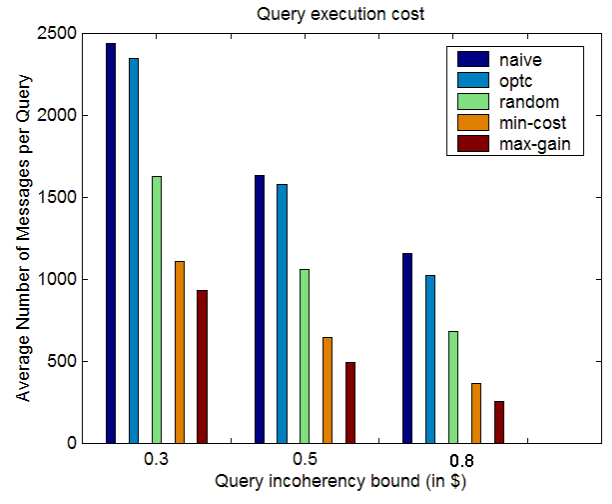

Figure 5: Performance evaluation of algorithms

\subsection{Effect of Algorithmic Parameters}

These set of experiments were performed to get an insight into various characteristics of our sub-query selection method which lead it to perform better compared to other options. We consider effects of three parameters on sub-query selection and, in turn on query performance: data dynamics, correlation between data dynamics and query satisfiability parameter.

\subsubsection{Effect of data dynamics}

In this set of experiments, we wanted to see whether there is any definite relationship between data dynamics and sub-query size in which that data item appears. In this experiment with 10 data items, 45 DAs were simulated such that each DA can disseminate a different set of 2 data items. Then 100 queries were created each with 3 data items. In the optimal query plan, each query will be executed with two sub-queries: one consisting of 2 data items and another with single data item (plan with three one item subqueries will be trivially inefficient). As the query has only 3 data items, only 3 such query plans are possible. We simulated all these options to get the best query plan. Figure 6 shows variation of average sub-query size in which a particular data item appears versus sumdiff value of the data item. We can see that if a data item is more dynamic, in the optimal plan, it is more likely to be part of larger sub-query. This is an important observation as it indicates that for efficient query evaluation more dynamic data items should be part of a larger sub-query. This phenomenon can be explained by the fact that by executing a query as a combination of sub-queries will always be more efficient compared to getting the data items independently. By combining 


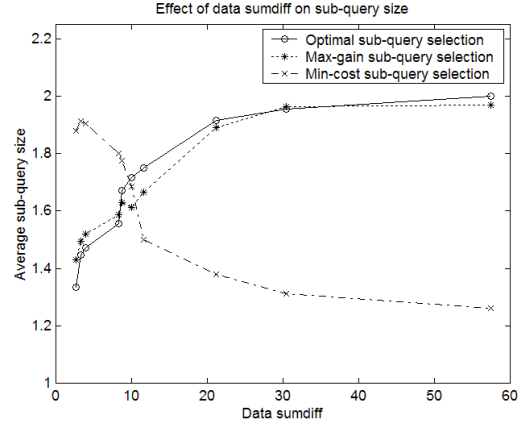

Figure 6: Effect of data sumdiff on sub-query size

more dynamic data items we are likely to gain more. For comparison we also show the curve for the sub-query selection based on max-gain algorithm. It can be seen that sub-query selection using max-gain is approximately same as that selected by the optimal solution. By using max-gain algorithm we achieve our objective of disseminating more dynamic data items as part of larger sub-queries. For the max-gain algorithm, similar results were obtained for larger query sizes as well. In comparison, in the min-cost algorithm most dynamic data item is more likely to be disseminated as single item query. This happens because the sumdiff value of a more dynamic data item will be high thus in each step of the min-cost algorithm (Figure 4), there is less chance of selecting a sub-query with more dynamic data item. Thus, it is very likely that the highly dynamic data item will be disseminated as a single item sub-query resulting in bad performance of the client query. Still the min-cost algorithm performs better compared to random algorithm as it tries to execute the query with lesser number of sub-queries.

\subsubsection{Effect of correlation between data dynamics}

To measure the effects of correlation between data dynamics (cosine similarity) on the query performance, we compared the query performance with the case when all the data items are assumed to be independent (i.e., $\rho=0$ ). For performing these experiments we constructed 10 synthetic data traces so that values of $\rho$ for various data item pairs were distributed uniformly between -1 and +1 . Then 45 DAs were simulated so that each DA can disseminate 2 data items. 100 queries were generated, each with 4 data items. In this case, each query will get executed with 2 sub-queries of 2 data items each. Combination of sub-queries will be decided based on correlation between data items (sumdiff values of all the data items were the same). Table 1 compares the

Table1: Effect of correlation on number of refreshes

\begin{tabular}{|c|c|c|}
\hline $\begin{array}{c}\text { Incoherency } \\
\text { Bound }\end{array}$ & $\begin{array}{c}\text { Avg. number of msgs } \\
\text { when } \rho \text { is considered }\end{array}$ & $\begin{array}{c}\text { Avg. number of msgs } \\
\text { when } \rho \text { is assumed to be } 0\end{array}$ \\
\hline 0.5 & 2301 & 2559 \\
\hline 0.8 & 1092 & 1215 \\
\hline 1.0 & 754 & 846 \\
\hline
\end{tabular}

results when cosine similarity is taken into account and when cosine similarity is assumed to be 0 for all data item pairs. It can be seen that by considering cosine similarity number of refreshes reduce by approximately $12 \%$. This result indicates that for subquery selection data dynamics may be more important factor than the cosine similarity between the data items.

\subsubsection{Effect of query satisfiability parameter}

To simulate the situation where selected aggregators may not be able to satisfy the query incoherency bounds, we modified the simulation set up used in Section 5.1 to set the minimum data incoherency bounds which DAs can satisfy to be between .015 and 0.04. Value of $\alpha$ was varied between $0-20$. The case $\alpha=0$ corresponds to the algorithm without dealing with the query satisfiability. Figure 7 shows query execution cost and number of unanswerable queries as the value of $\alpha$ is varied. As shown in the figure as the value of $\alpha$ is increased, percentage of the unsatisfied queries decreased for various values of query incoherency bounds.

Due to changed data incoherency bounds of DAs, we found that $20 \%$ of queries can not be satisfied even by the data aggregators with tightest data incoherency bounds. Thus, while presenting the results, we remove those queries. At the query incoherency bound of $\$ 0.8,40 \%$ are queries can not be satisfied by the optimally

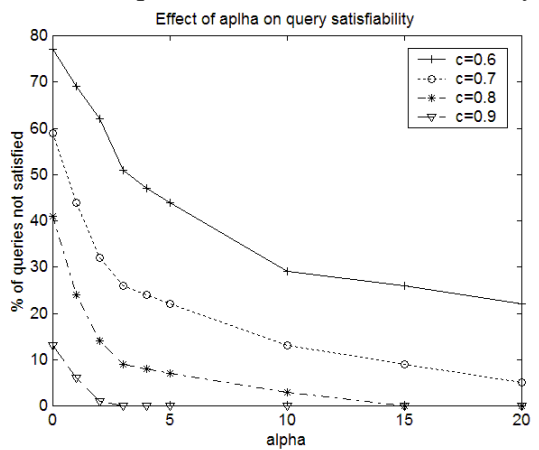

Figure 7: Effect of $\alpha$ on query satisfiability

selected data aggregators but as we increase the value of $\alpha$ to 10 , only $3 \%$ queries are unanswered. Such a value can be chosen to balance the performance and satisfiability of queries. For example, a dynamic CDN may aim at query satisfiability of $95 \%$ for a given distribution of query incoherency bounds. If at any time query satisfiability is below the target, value of $\alpha$ can be increased whereas in case of over achieving the target, the value of $\alpha$ can be decreased to improve the query performance.

\subsubsection{Summary of performance results}

Following features of the query planning algorithm improve performance:

* Dividing the query into sub-queries and executing them at specifically chosen data aggregators.

* Deciding the query plan using data sumdiff based mechanism specifically by maximizing sub-query gains.

* Including more dynamic data as part of a larger sub-query.

We also showed that the max-gain algorithm is very close to the optimal algorithm in selecting sub-queries based on data dynamics.

\section{RELATED WORK}

Various mechanisms for efficiently maintaining incoherency bounded aggregation queries over continuously changing data items are proposed in the literature $[11,12,16]$. Our work distinguishes itself by being sub-query based evaluation to minimize number of refreshes. In [11], authors propose using data filters at the sources; instead we assign incoherency bounds to sub-queries which reduce the number of refreshes for query evaluation, as explained in Section 
5. Further, we propose that more dynamic data items should be executed as part of larger sub-query.

In [22], authors present technique of reorganizing a data dissemination network when client requirements change. Instead, we try to answer the client query using the existing network. Reorganizing aggregators is a longer term activity whereas query planning can be done for short as well as long running queries on more dynamic basis.

Pull based data dissemination techniques, where clients or data aggregators pull data items such that query requirements are met, are described in $[9,16]$. For minimizing the number of pulls, both model the individual data items and predict data values. In comparison, we consider the situation where different sub-queries, involving multiple data items, can be evaluated at different nodes. Further, incoherency bound is applied over the sub-query rather than to individual data items, leading to efficient evaluation of the query. Spatial and temporal correlations between sensor data are used to reduce data refresh instances in $[17,18]$. We also consider correlation in terms of cosine similarity between data items, but we use it for dividing client query into sub-queries. Our work can be extended by using temporal and spatial properties of data items for predicting their correlation measures. A method of assigning clients data queries to aggregators in a content distribution network is given in [12]. We do for client queries consisting of multiple data items what [12] does for client requiring individual data items.

\section{CONCLUSIONS}

This paper presents a cost based approach to minimize the number of refreshes required to execute an incoherency bounded continuous query. For optimal execution we divide the query into sub-queries and evaluate each sub-query at a chosen aggregator. Performance results show that by our method the query can be executed using less than one third the messages required for existing schemes. Further we showed that by executing queries such that more dynamic data items are part of a larger sub-query we can improve performance. Our method of query execution can be implemented using schemes similar to that used in CDNs. Our query cost model can also be used for other purposes such as load balancing various aggregators, optimal query execution plan at an aggregator node, etc. Using the cost model for other applications and developing the cost model for more complex queries is our future work.

Acknowledgement: We would like to thank Venkatesan Chakravarthy and Vinayak Pandit for helpful discussion on various algorithms.

\section{REFERENCES}

[1] A. Davis, J. Parikh and W. Weihl. Edge Computing: Extending Enterprise Applications to the Edge of the Internet. WWW 2004

[2] D. VanderMeer, A. Datta, K. Dutta, H. Thomas and K. Ramamritham. Proxy-Based Acceleration of Dynamically Generated Content on the World Wide Web. ACM Transactions on Database Systems (TODS) Vol. 29, June 2004.

[3] J. Dilley, B. Maggs, J. Parikh, H. Prokop, R. Sitaraman and B. Weihl. Globally Distributed Content Delivery, IEEE Internet Computing Sept 2002.
[4] S. Rangarajan, S. Mukerjee and P. Rodriguez. User Specific Request Redirection in a Content Delivery Network, $8^{\text {th }}$ Intl. Workshop on Web Content Caching and Distribution (IWCW), 2003.

[5] S. Shah, K. Ramamritham, and P. Shenoy. Maintaining Coherency of Dynamic Data in Cooperating Repositories. VLDB 2002.

[6] Dynamai: Caching Technology for Dynmaic Content Revealed. www.infoworld.com/articles.

[7] D. S. Hochbaum. Approximation algorithms for the set covering and vertex cover problems. SIAM Journal on Computing, vol. 11 (3), 1982.

[8] Zongming Fei. A Novel Approach to Managing Consistency in Content Distribution. WCW 2001

[9] R. Gupta, A. Puri, and K. Ramamritham. Executing Incoherency Bounded Continuous Queries at Web Data Aggregators. WWW 2005.

[10] Optimized Execution of Continuous Queries, APS 2006, www.cse.iitb.ac.in/ grajeev/APS06.PDF

[11]C. Olston, J. Jiang, and J. Widom. Adaptive Filter for Continuous Queries over Distributed Data Streams. SIGMOD 2003.

[12] S. Shah, K. Ramamritham, and C. Ravishankar. Client Assignment in Content Dissemination Networks for Dynamic Data. VLDB 2005.

[13] NEFSC Scientific Computer System http://sole.wh.whoi.edu/ jmanning//cruise/serve1.cgi

[14] Query cost model validation for sensor data. www.cse.iitb.ac.in/ ravivj/BTP06.pdf.

[15]D. S. Hochbaum. Approximation algorithms for the set covering and vertex cover problems. SIAM Journal on Computing, vol. 11 (3), 1982.

[16] S. Zhu and C. Ravishankar. Stochastic Consistency and Scalable Pull-Based Caching for Erratic Data Sources. VLDB 2004.

[17] D. Chu, A. Deshpande, J. Hellerstein, W. Hong. Approximate Data Collection in Sensor Networks using Probabilistic Models. ICDE 2006.

[18] A. Deshpande, C. Guestrin, S. R. Madden, J. M. Hellerstein, and W. Hong. Model-Driven Data Acquisition in Sensor Networks. VLDB, 2004.

[19] Pearson Product moment correlation coefficient. http://www.nyx.net/ tmacfarl/STAT_TUT/correlat.ssi /

[20] Lam, W. and Ho, C.Y. Using a Generalized Instance Set for Automatic Text Categorization. SIGIR, 1998.

[21] G. Cormode and M. Garofalakis. Sketching Streams through the Net: Distributed Approximate Query Tracking. VLDB 2005.

[22] S. Agrawal, K. Ramamritham and S. Shah. Construction of a Temporal Coherency Preserving Dynamic Data Dissemination Network. RTSS 2004. 